\title{
Effects of Allicin on the Formation of Pseudomonas aeruginosa Biofilm and the Production of Quorum-Sensing Controlled Virulence Factors
}

\author{
LIN LIHUA ${ }^{2,3 \dagger}$, WANG JIANHUI ${ }^{1, \dagger}$, YU JIALIN ${ }^{1,2,3 *}$, LI YAYIN ${ }^{2,3}$ and LIU GUANXIN ${ }^{3,4}$ \\ ${ }^{1}$ Diagnostic and Treatment Center of Neonatology, the Children's Hospital \\ of Chongqing Medical University, Chongqing, China \\ ${ }^{2}$ Ministry of Education Key Laboratory of Child Development and Disorders, Chongqing, China \\ ${ }^{3}$ Key Laboratory of Pediatrics in Chongqing, Chongqing, China \\ ${ }^{4}$ Chongqing International Science and Technology Cooperation Center for Child Development \\ and Disorders, the Children's Hospital of Chongqing Medical University, Chongqing, China
}

Submitted 13 July 2012, revised 13 April 2013, accepted 15 July 2013

\section{Abstract}

\begin{abstract}
The Gram-negative Pseudomonas aeruginosa bacterial pathogen is reputed for its resistance to multiple antibiotics, and this property is strongly associated with the development of biofilms. Bacterial biofilms form by aggregation of microorganisms on a solid surface and secretion of an extracellular polysaccharide substances that acts as a physical protection barrier for the encased bacteria. In addition, the $P$. aeruginosa quorum-sensing system contributes to antibiotic resistance by regulating the expression of several virulence factors, including exotoxin A, elastase, pyoverdin and rhamnolipid. The organosulfur compound allicin, derived from garlic, has been shown to inhibit both surface-adherence of bacteria and production of virulence factors. In this study, the effects of allicin on P. aeruginosa biofilm formation and the production of quorum-sensing controlled virulence factors were investigated. The results demonstrated that allicin could inhibit early bacterial adhesion, reduce EPS secretion, and down-regulate virulence factors' production. Collectively, these findings suggest the potential of allicin as a therapeutic agent for controlling P. aeruginosa biofilm.
\end{abstract}

Ke y w o r d s: Pseudomonas aeruginosa, allicin; antibiotic resistance, biofilm, virulence factor

\section{Introduction}

Pseudomonas aeruginosa is a prevalent opportunistic bacterial pathogen that commonly infects immunocompromised patients and colonizes indwelling catheters (Bartoszko-Tyczkowska et al., 2008). It is also one of the leading causes of nosocomial infections throughout of the world, causing severe and life-threatening pulmonary infection (Koulenti et al., 2009). P. aeruginosa is reputed for its ability to rapidly develop resistance to multiple antibiotics, a characteristic that is particularly challenging to healthcare staff. The antibiotic-resistant property of $P$. aeruginosa has been extensively studied and found to involve two primary mechanisms: active efflux pumps that expel antibiotics from the bacteria, and surface-bound bacterial biofilms (Prithiviraj et al., 2005). Biofilms form on solid surfaces when microorganisms, such as bacteria or fungi, aggregate and reach a certain threshold that facilitates intercellular signaling events, a process known as quorum-sensing
(QS). QS stimulates localized bacteria to produce and secrete a polymeric matrix, which consists of various secreted proteins, nucleic acids, polysaccharides and minerals. This extracellular matrix acts to encase the bacteria, providing a physical barrier against antimicrobial agents (Costerton, 2001). In addition, the bacteria inside the biofilm enter a quiescent state, and this non-metabolically active state renders the bacteria insensitive to any antimicrobial agents that may have penetrated the biofilm (Drenkard, 2003).

The QS system also regulates many biological behaviors of the mature biofilm. For example, the expression of several extracellular virulence factors, including exotoxin A, elastase, pyoverdin and rhamnolipid (Kirisits and Parsek, 2006; Rumbaugh et al., 2000), is regulated by QS. These factors are critical to establishing infection in the host and contribute antibiotic resistance properties, as well; therefore, inhibition of the QS system is believed to be a promising strategy to attenuate production of virulence factors and protect against infection.

* Corresponding author: Yu Jialin, No. 136, Zhongshan Second Street, Yuzhong District, Chongqing 400014, China. phone: +86 023-63635567; e-mail: yujialin486@sohu.com

${ }^{\dagger}$ These two authors contributed equally to this work and share the first author together. 
The organosulfur compound allicin which is derived from garlic, has antibacterial properties, and has been reported to effectively inhibit Staphylococcus epidermis, another common nosocomial pathogen (PérezGiraldo et al., 2003) and Aggregatibacter actinomycetemcomitans, the key etiologic agent of the severe form of localized aggressive periodontitis (Velliyagounder et al., 2012). At concentrations below the minimum inhibitory concentration (MIC), allicin has been shown to prevent adherence of S.epidermis to polystyrene microtiter plates (Pérez-Giraldo et al., 2003), suggesting a potential therapeutic role for allicin as a biofilm inhibitor. A previous study of allicin's effects on P. aeruginosa determined that this agent can specifically inhibit QS-related gene expression (Bjarnsholt et al., 2005). This finding led to the hypothesis that allicin may act as an anti-biofilm agent against $P$. aeruginosa. Therefore, this study was designed to investigate the effects of allicin on $P$. aeruginosa biofilm formation, including the secretion of extracellular polysaccharide substances (EPS) and induced expression of QS-mediated virulent factors.

\section{Experimental}

\section{Materials and Methods}

Strains. P.aeruginosa strains used in this study were wild-type ATCC 27853 PAO1 (American Type Culture Collection, Manassas, VA, USA) and green fluorescent protein (GFP)-transformed P. aeruginosa PAO1. Bacteria were cultured in Luria-Bertani (LB) medium ( $1 \%$ tryptone, $0.5 \%$ yeast extract, $0.5 \% \mathrm{NaCl}$, $\mathrm{pH} 7.5$ ), peptone-tryptic soy broth (PTSB; 5\% peptone, $0.25 \%$ tryptic soy broth), and tryptic soy broth (TSB; 3\%). All of the broths were obtained from SigmaAldrich (St. Louis, MO, USA). Meanwhile, the Super Optimal Broth (SOB) medium ( $2 \%$ tryptone, $0.5 \%$ yeast extract, $0.05 \% \mathrm{NaCl}, 0.0186 \% \mathrm{KCl}, \mathrm{pH} 7.0)$ and the SOB with catabolite repression (SOC) medium $(50 \mathrm{~mL}$ SOB medium with added $1 \mathrm{ml}$ glucose at $1 \mathrm{~mol} / \mathrm{l}$ ) were used during the construction of GFP-transformed P. aeruginosa PAO1.

Construction of GFP-transformed P. aeruginosa PAO1 (according to the method described by Smith and Iglewski, 1989). Overnight culture of strain P. aeruginosa $\mathrm{PAO} 1$, at $\mathrm{OD}_{600}$ of 0.7 , were grown in $50 \mathrm{~mL} \mathrm{SOB}$ medium with vigorous shaking at $37^{\circ} \mathrm{C}$. The cells were harvested by centrifugation at $22500 \times g$ for $15 \mathrm{~min}$ at $2{ }^{\circ} \mathrm{C}$, washed in the same volume of $0.3 \mathrm{~mol} / \mathrm{l}$ sucrose, then washed in 0.5 volume of wash medium and in 0.2 volume, re-centrifuged, and finally resuspended in 0.01 volumes of $0.3 \mathrm{~mol} / 1$ sucrose $\left(1 \times 10^{11} \mathrm{cfu} / \mathrm{ml}\right)$. The map of pGFPuv plasmid was shown in Fig. 1, and the promoter is lacZ. One hundred $\mu \mathrm{L}$ aliquots of cell suspension were mixed with 50 ng pGFPuv plasmid, and transferred to chilled $0.1 \mathrm{~cm}$ gap cuvettes. Following delivery of the pulse (voltage $1.5 \mathrm{kV}$, capacitance $25 \mu \mathrm{F}$, resistance $200 \Omega$ ), the cells were mixed with $800 \mu \mathrm{l} \mathrm{SOC}$ medium and shaken for $1 \mathrm{~h}$ at $37^{\circ} \mathrm{C}$. One hundred $\mu \mathrm{L}$ aliquots of suspension were then cultured in the screening medium for $16 \mathrm{~h}$ at $37^{\circ} \mathrm{C}$, the bacterial colony exhibiting green bright-green fluorescence was the GFP-transformed P. aeruginosa PAO1.

Susceptibility testing methods. The MIC of P. aeruginosa strains was determined by the standard microdilution method using Mueller-Hinton broth and custom-made 96-well plates. The procedure followed the NCCLS guidelines (Clinical and Laboratory Standards Institute, 2007).

Biofilm formation assay. The ability of GFP-transformed P. aeruginosa PAO1 to form biofilm on abiotic surfaces was investigated by standard fluorescence assay. Briefly, overnight cultures of strain GFP-transformed P.aeruginosa PAO1 were grown in 96-well polyvinyl chloride (PVC) microtiter plates at $37^{\circ} \mathrm{C}$ in LB medium. For experimental groups, allicin was added at the concentration of 10 or $128 \mathrm{~g} / \mathrm{ml}$ for $1,3,5$, 6 , and $9 \mathrm{~h}$. The relative adhesion ratio of bacteria was calculated according to the fluorescence detected at an excitation wavelength of $485 \mathrm{~nm}$ and an emission wavelength of $528 \mathrm{~nm}$ on a micro plate reader (Biotek Synergy HT; Hamilton, Reno, NV, USA). Overnight cultures of GFP-transformed P. aeruginosa PAO1, at $\mathrm{OD}_{600}$ of 0.5 , were seeded onto coverslips in 24-well PVC microtiter plates and grown at $37^{\circ} \mathrm{C}$ in $\mathrm{LB}$ medium with or without allicin $(10$ or $128 \mu \mathrm{g} / \mathrm{ml})$ for $6 \mathrm{~h}$. The coverslips were then washed with phosphate buffered saline (PBS) and examined by fluorescence microscopy.

Extraction and quantification of EPS (according to the method described by Akiyama et al., 2002; Petit et al., 2007). Overnight cultures of strain GFP-transformed P. aeruginosa $\mathrm{PAO} 1$, at $\mathrm{OD}_{600}$ of 0.5 , were grown on coverslips in 24 -well $\mathrm{PVC}$ microtiter plates at $37^{\circ} \mathrm{C}$ in LB medium for $12 \mathrm{~h}$. The $\mathrm{pH}$ of the medium was adjusted to 7.0 with $1 \mathrm{~mol} / 1 \mathrm{NaOH}$. Bacterial cultures were grown with and without added allicin at a concentration of 10 or $128 \mu \mathrm{g} / \mathrm{ml}$ for $6 \mathrm{~h}$. The coverslips were then washed with PBS and immersed in 3\% methanol for $15 \mathrm{~min}$. After washing with PBS, the coverslips were immersed in FITC-ConA ( $50 \mu \mathrm{g} / \mathrm{ml})$ for $15 \mathrm{~min}$ at room temperature, and observed by fluorescence microscopy, with maximum emission at $488 \mathrm{~nm}$. An overnight culture of GFP-transformed P. aeruginosa PAO1 in LB medium was diluted 100 -fold into $250 \mathrm{ml} \mathrm{LB}$ medium, and allicin (at 10 or $128 \mathrm{~g} / \mathrm{ml}$ ) was added and incubated for $5 \mathrm{~h}$ at $37^{\circ} \mathrm{C}$ with shaking. The EPS was obtained by three rounds of centrifugation at $10000 \times g$ for $30 \mathrm{~min}$ each. The precipitate was air-dried for $4 \mathrm{~h}$ and then added with alcohol overnight. The precipitate was 


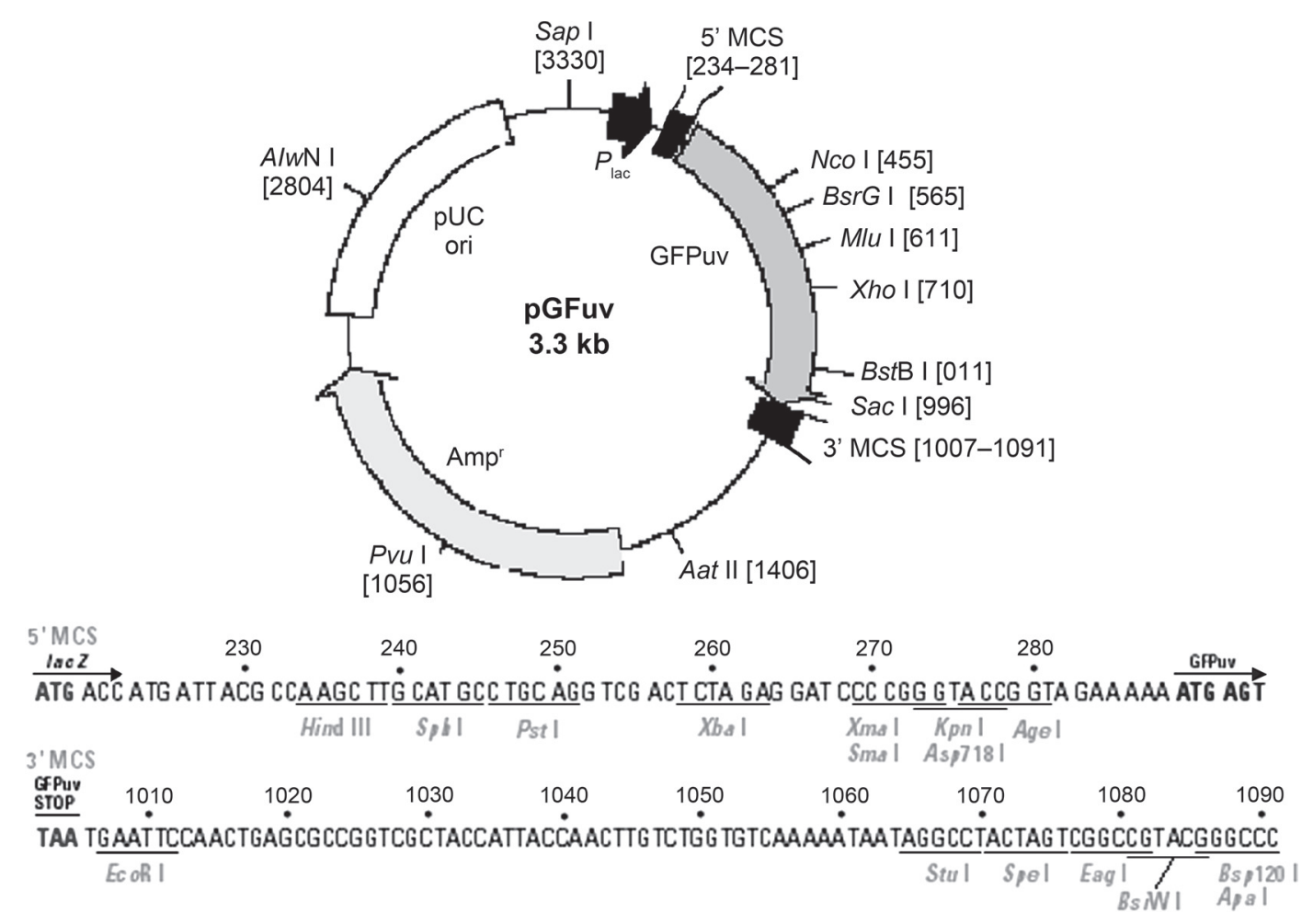

Fig. 1. The map of pGFPuv plasmid. The promoter is lacZ.

then dissolved in $2 \mathrm{ml}$ of distilled water. When treated with phenol and concentrated sulfuric acid, the purified saccharides produced an orange-yellow color that was stable over time. This method has been reported to quantify the EPS content (DuBois et al., 1956).

Biofilm assay (Beyenal et al., 2004). The influence of allicin on biofilm formation of GFP-transformed P. aeruginosa $\mathrm{PAO} 1$ was evaluated by confocal laser scanning microscopy (CLSM). To quantify the biofilm structure, Image Structure Analyzer (ISA) software was used. Each assay was performed in quadruplicate and repeated three or more times. Overnight cultures of strain GFP-transformed P. aeruginosa $\mathrm{PAO} 1$, at $\mathrm{OD}_{600}$ of 0.5 , were grown on coverslips in 24-well PVC microtiter plates at $37^{\circ} \mathrm{C}$ in $\mathrm{LB}$ medium for $1,3,7 \mathrm{~d}$, and medium was changed on alternate days. Then, coverslips were grown with and without added allicin at 10 or $128 \mu \mathrm{g} / \mathrm{ml}$ for $6 \mathrm{~h}$, and the coverslips were washed with PBS before being immersed in SYTO9/PI (L13152; Molecular Probes, Eugene, OR, USA) and incubated for $15 \mathrm{~min}$ at room temperature in the dark. After washing, the coverslips were observed with CLSM. Images were obtained with an LSM 510 confocal microscope (Leica Co., Ltd., Solms Germany). The confocal microscope was equipped with a $100 \times, 1.3$-numerical aperture (NA) oil immersion, phase-contrast lens. When used in conjunction with confocal laser technology, the highNA lens has the potential to produce images with sub200-nm horizontal resolution in the XY axis and with reduced defocused information from XY materials. An argon laser with maximum emission at $488 \mathrm{~nm}$ was used as the excitation source for the fluorescent probe SYTO9/PI. CLSM data was analyzed using the ISA software program, and quantitative parameters describing biofilm physical structure were extracted from three-dimensional (3D) CLSM images. This software describes parameters as follows: three-dimensional biofilm average diffusion distance (ADD), maximum diffusion distances (MDD), fractal dimension, average run lengths (in $\mathrm{X}, \mathrm{Y}$ and $\mathrm{Z}$ directions), area porosity (AP), textural entropy (TE), energy, homogeneity and heterogeneity, size, and morphology of biomass.

Growth curves. The effect of allicin on cell proliferation was determined by monitoring the strain GFPtransformed P. aeruginosa PAO1 growth curve. Briefly, an overnight culture (in LB medium) of GFP-transformed P. aeruginosa PAO1 was diluted 100-fold into $250 \mathrm{ml}$ of LB medium. The culture was then divided into two groups: LB medium alone (control) and allicin treatment at $128 \mu \mathrm{g} / \mathrm{ml}$ (experimental). The $\mathrm{OD}_{600}$ was monitored at $1 \mathrm{~h}$ intervals up to $24 \mathrm{~h}$.

Exotoxin A assay (Kozak and Saelinger, 1988; Shigematsu et al., 2007). Bacteria were grown in a dialysate of trypticase soy broth (TSBD), consisting of TSB with $1 \%$ glycerol and $50 \mathrm{mM}$ monosodium glutamate, at $32^{\circ} \mathrm{C}$ with shaking for $18 \mathrm{~h}$. The culture was then divided into two groups: TSBD medium alone (control) and allicin treatment at $128 \mu \mathrm{g} / \mathrm{ml}$ (experimental). The cultures were grown for $5.5 \mathrm{~h}$ and then the cells were harvested by centrifugation at $10000 \times \mathrm{g}$ for $30 \mathrm{~min}$ at $4^{\circ} \mathrm{C}$, 
and the supernatant fluid (spent medium) was purified by filtration through a membrane filter $(0.45 \mathrm{~m}$ porosity). Spent media were kept on ice throughout all procedures, and samples were stored at -20 or $-70^{\circ} \mathrm{C}$. Enzyme-linked immunosorbent assay (ELISA) was used for the quantification of exotoxin A.

LasB elastolytic assay (according to Ohman et al., 1980). Overnight cultures of strain GFP-transformed P. aeruginosa $\mathrm{PAO} 1$ were grown in $50 \mathrm{~mL}$ PTSB medium at $37^{\circ} \mathrm{C}$ with shaking. The cultures were then diluted in PTSB medium and allowed to grow to an $\mathrm{OD}_{600}$ of 1.5 (middle of logarithmic phase). The cells were separated from the growth medium by centrifugation at $10000 \times \mathrm{g}$ for $30 \mathrm{~min}$, and re-cultured in PTSB medium at $37^{\circ} \mathrm{C}$ with shaking for $15 \mathrm{~h}$. At this point, the culture was divided into $10 \mathrm{~mL}$ aliquots and an additional $1 \mathrm{ml}$ of allicin (or media control) was added to a final concentration of $128 \mu \mathrm{g} / \mathrm{ml}$ and the mixtures incubated at $37^{\circ} \mathrm{C}$ for $6 \mathrm{~h}$. The supernatants were collected by centrifugation and filtered through an $0.45 \mu \mathrm{m}$ nylon filter. The elastolytic activity of the PTSB medium culture supernatants was determined by staining with elastin congo red (ECR; Sigma-Aldrich). Briefly, a $50 \mathrm{~mL}$ aliquot of supernatant was added to $1 \mathrm{ml}$ of ECR buffer $(100 \mathrm{mM}$ Tris, $1 \mathrm{mM} \mathrm{CaCl}$, pH 7.2) containing $20 \mathrm{mg}$ of ECR. This mixture was then incubated with shaking at $37^{\circ} \mathrm{C}$ for $15 \mathrm{~h}$. Insoluble ECR was removed by centrifugation, and the absorption of the supernatant was measured at $495 \mathrm{~nm}$. Cell-free LB medium alone and LB medium with plant extracts were used as negative controls.

Pyoverdin assay (Cox and Adams, 1985). The pyoverdin assay was adapted from the methods of Cox and Adams. First, the culture was divided into two groups: LB medium (control) and allicin at $128 \mu \mathrm{g} / \mathrm{ml}$ (experimental). The LB medium culture supernatant was diluted 10-fold in Tris- $\mathrm{HCl}$ buffer ( $\mathrm{pH} 7.4$ ), and $100 \mu \mathrm{l}$ aliquots were added to 96 -well microtiter plates on ice. The relative concentration of pyoverdin was calculated based on the fluorescence of the supernatant measured at an excitation wavelength of $405 \mathrm{~nm}$ and an emission wavelength of $465 \mathrm{~nm}$ on a Biotek Synergy HT instrument. Activity was expressed in relative fluorescence units. Although pyoverdin was considered to be a marker of QS, a drop in production may be due to an indirect effect of $\mathrm{pH}$ or iron concentration changes. To eliminate the chance of false-positive results, the solution $\mathrm{pH}$ was monitored throughout the experiment (Harjai et al., 2005).

Rhamnolipid assay. Bacterial cultures were grown with and without allicin. The culture broth of P. aeruginosa was processed as follows: bacterial cells were removed from the biosurfactant-containing medium by centrifugation at $10000 \times \mathrm{g}$ for $30 \mathrm{~min}$ at $4^{\circ} \mathrm{C}$. The $\mathrm{pH}$ of the supernatant was adjusted to $2.0 \pm 0.5$ with $1 \mathrm{M} \mathrm{HCl}$ to reduce biosurfactant solubility. The biosurfactant was extracted by three rounds of washing with an equal volume of chloroform-methanol (2:1). The solvent was then evaporated and the resultant thick yellowish product was dissolved in $0.05 \mathrm{M} \mathrm{NaHCO}_{3}$. Rhamnolipids in the supernatant were quantified as rhamnose concentration using $\mathrm{H}_{2} \mathrm{SO}_{4}$ anthrone. The rhamnolipid concentration was calculated by multiplying rhamnose values by a coefficient of 3.4 (Benincasa et al., 2004).

Statistical analysis. All experiments were performed independently in triplicate with pooled samples of biological replicates, and data are presented as mean standard deviation (SD). Intergroup differences were analyzed by student's $t$ test using the SPSS 17.0 statistical software package (Chicago, IL, USA). A $p$-value less than 0.05 was considered significant.

\section{Results}

Effects of allicin on P. aeruginosa biofilm adhesion. The GFP-expressing bacterial colonies were found in both the saline control group and allicin treatment group by fluorescence microscopy (Fig. 2). After treatment with allicin at the concentration of $128 \mu \mathrm{g} / \mathrm{ml}$, the
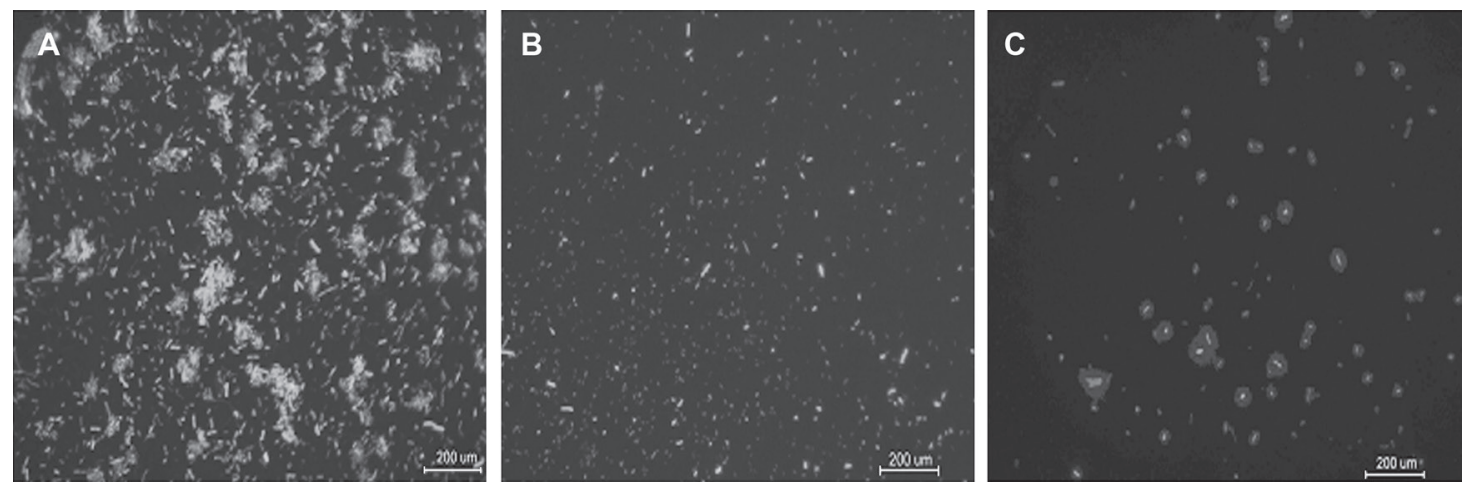

Fig. 2. Adhesion of pGFP transformed P. aeruginosa PAO1 to form biofilms.

Bacterial adhesion was reduced and the bacterial distribution became looser in response to allicin treatment. P. aeruginosa treated with saline (A, control), allicin at $10 \mu \mathrm{g} / \mathrm{ml}$ (B), or allicin at $128 \mu \mathrm{g} / \mathrm{ml}$ (C). 


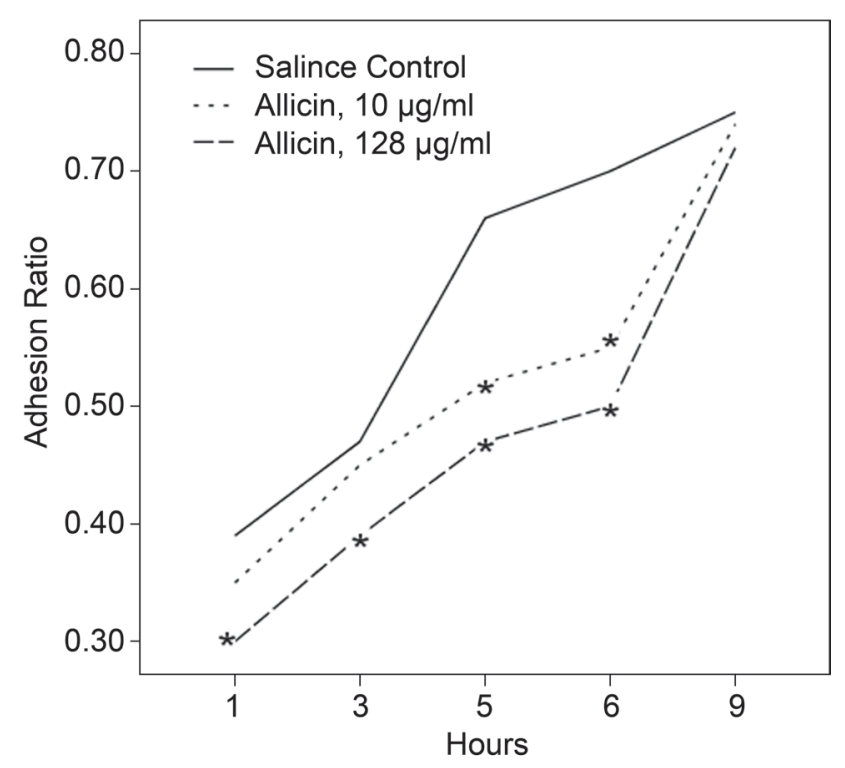

Fig. 3. Adhesion ratio of each group over time. ${ }^{*} p<0.05$, determined by Student's $t$-test when compared with the saline control group.

surface-adherence of bacteria was significantly reduced and the bacterial distribution became more scattered, as compared with that observed for the saline control group. At the lower concentration of $10 \mu \mathrm{g} / \mathrm{ml}$, allicin treatment produced a less robust effect. The adhesion ratio defined by the percentage of adhering bacteria to total number of bacteria for untreated wild-type $P$. aeruginosa $\mathrm{PAO} 1$ increased with the time passed (Fig. 3). After allicin treatment at the concentration of $128 \mu \mathrm{g} / \mathrm{ml}$ for $6 \mathrm{~h}$, the adhesion ratio decreased from $0.70 \pm 0.03$ to $0.50 \pm 0.01$ ( $t=15.014, p<0.05)$; however, after $9 \mathrm{~h}$ of allicin treatment, the observed effect on adhesion was lost.

Effects of allicin on EPS production of biofilm. The EPS in biofilm was detected as green fluorescence on fluorescence microscope (Fig. 4). The production of EPS was found to be increased with the time passed, and decreased by allicin treatment in a concentration- dependent manner, as compared with the saline control group. Although the dry weight of bacteria among the different groups had been almost equal at the onset of experiments, the total production of EPS was remarkably less in reactions treated with allicin, and the reduction in EPS content appeared to be dose-dependent for allicin (Table I).

Table I

Quantification of dry weight of bacteria and EPS in response to allicin treatment $(\mathbf{x} \pm \mathrm{SD})$

\begin{tabular}{|l|c|c|c|}
\hline \multicolumn{1}{|c|}{ Group } & $\begin{array}{c}\text { Dry weight } \\
\text { of bacteria, } \\
\mu \mathrm{g}\end{array}$ & EPS, $\mu \mathrm{g}$ & $\begin{array}{c}\text { EPS, } \mu \mathrm{g} / \text { Dry } \\
\text { weight of bac- } \\
\text { teria, } 100 \mu \mathrm{g}\end{array}$ \\
\hline Saline control & $30.81 \pm 0.36$ & $602.66 \pm 21.94$ & $195.57 \pm 5.36$ \\
\hline Allicin, $10 \mu \mathrm{g} / \mathrm{mL}$ & $30.45 \pm 0.95$ & $217.10 \pm 8.68^{*}$ & $71.29 \pm 2.20^{*}$ \\
\hline Allicin, $128 \mu \mathrm{g} / \mathrm{mL}$ & $30.37 \pm 1.00$ & $181.19 \pm 1.59^{*}$ & $59.70 \pm 1.47^{*}$ \\
\hline
\end{tabular}

${ }^{*} p<0.05$, determined by Student's $t$-test when compared with the saline control group.

Effect of allicin on biofilm development. The CLSM images of the flow chamber cultivations were acquired on 1, 3, and $7 \mathrm{~d}$ at random positions (Fig. 5). A red signal indicated dead bacteria and a green signal indicated live bacteria. By day 7, the GFP-transformed P. aeruginosa $\mathrm{PAO} 1$ had formed a mature structure that resembled mushrooms with rough surfaces, and showed a huge number of both dead and live bacteria. In the allicin-treated group (at $10 \mu \mathrm{g} / \mathrm{ml}$ ), the biofilm was thinner and looser in structure. Allicin treatment at the higher concentration of $128 \mu \mathrm{g} / \mathrm{ml}$ produced similar but more extensive effects on biofilm development.

Quantitative analysis of biofilm structure by ISA software. The ISA software was used to perform quantitative analysis of biofilm architecture. In the control group, the biofilm thickness was found to have increased over time, from $12.94 \mu \mathrm{m}$ on day 1 , to $23.98 \mu \mathrm{m}$ on day 3 , and then to $28.83 \mu \mathrm{m}$ on day 7 . The AP, on the
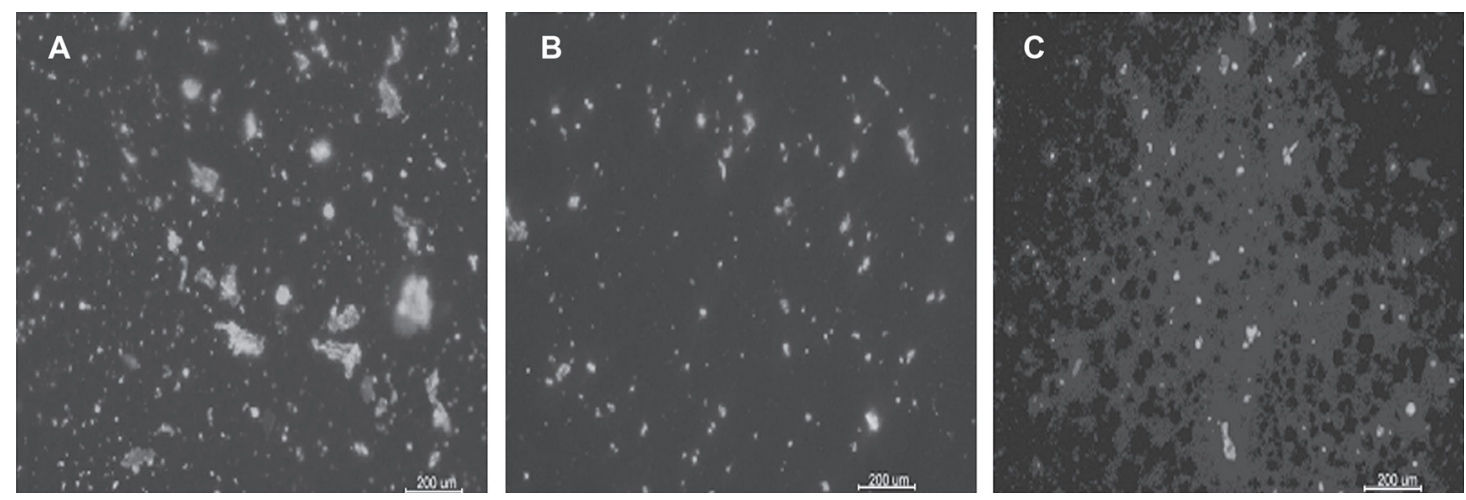

Fig. 4. EPS in bacterial biofilms.

As evidenced by FITC-ConA labeling $(\times 400)$, production of EPS was decreased after treatment with allicin, in a concentration-dependent manner. P. aeruginosa treated with saline (A, control), allicin at $10 \mu \mathrm{g} / \mathrm{ml}$ (B), or allicin at $128 \mu \mathrm{g} / \mathrm{ml}$ (C). 

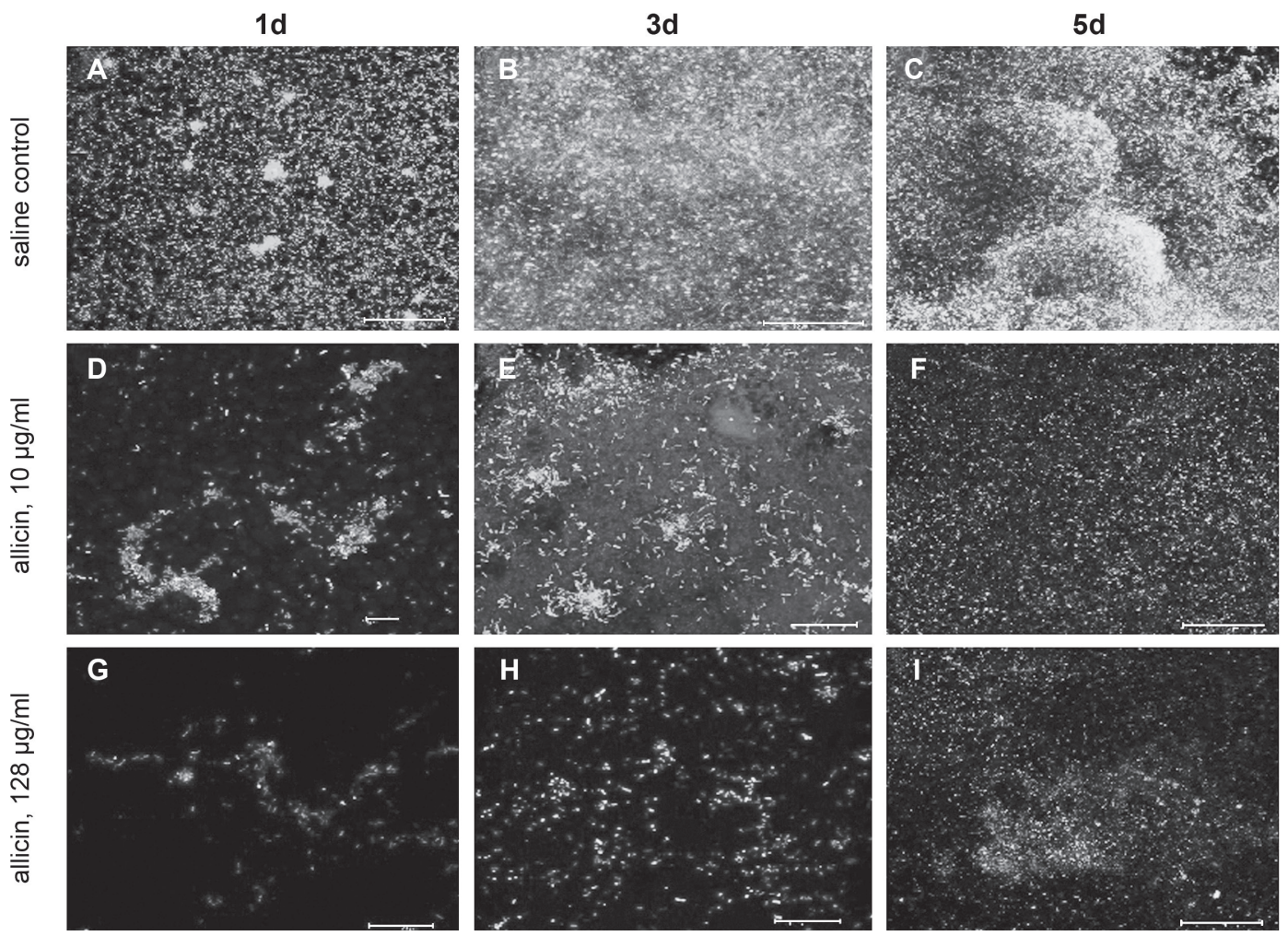

Fig. 5. CLSM images of immature and mature biofilm models treated with allicin.

Allicin treatment caused the biofilm to be thinner and looser in structure. (A-C) Saline control group and (D-I) allicin treatment groups (D, H: $10 \mu \mathrm{g} / \mathrm{ml}$; E, I: $128 \mu \mathrm{g} / \mathrm{ml}$ ), at day 1 (A, D, G), 3 (B, E, H), and 7 (C, F, I).

Dead bacteria are shown in red and live bacteria in green. Biofilm matured on day 7.
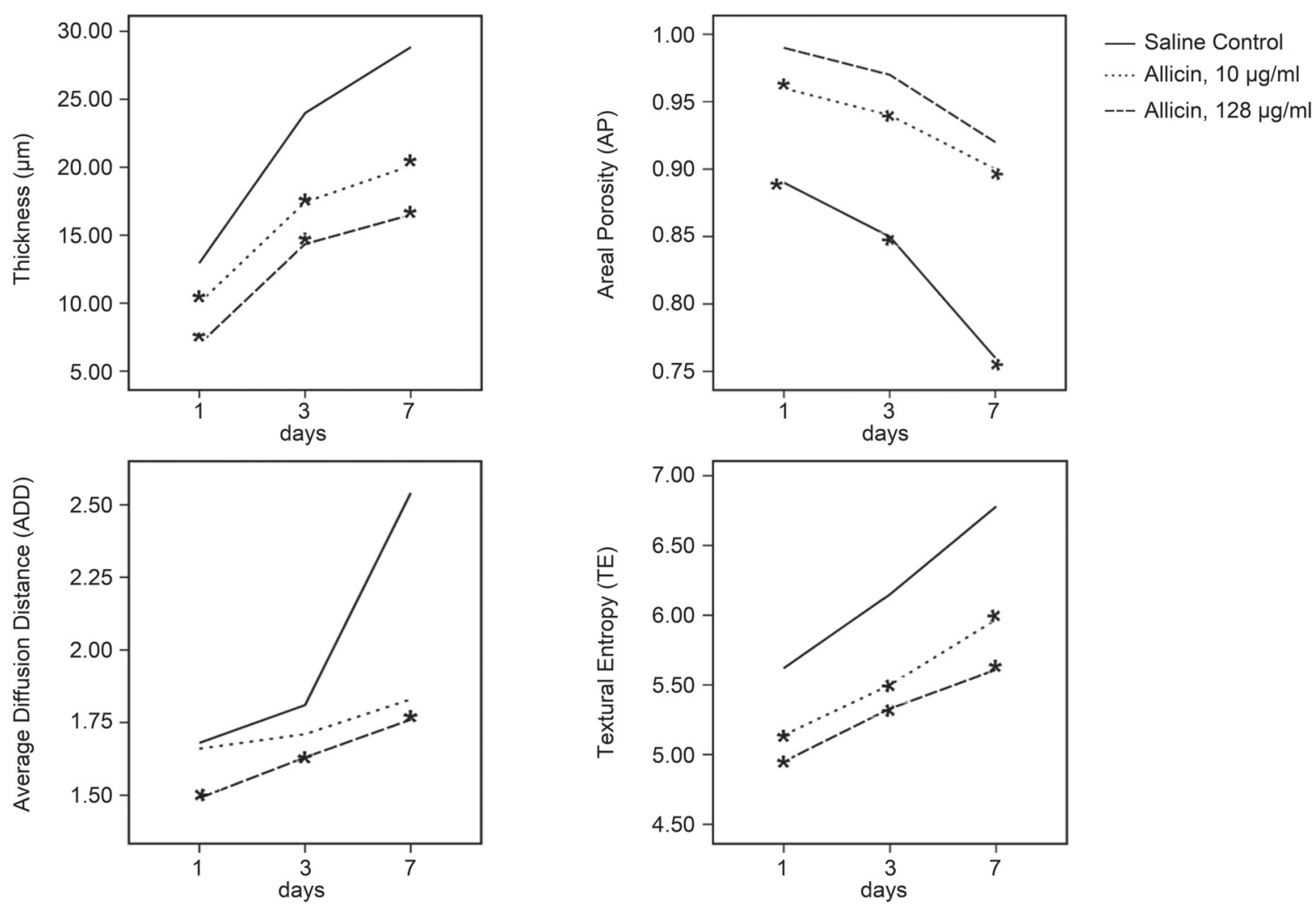

Fig. 6. Effect of allicin on some biofilm parameters, as calculated by ISA software.

Common parameters of biofilm architecture: mean thickness, areal porosity (AP), average diffusion distance (ADD), and biofilm thickness. ${ }^{*} p<0.05$, determined by Student's $t$-test when compared with the saline control group. 


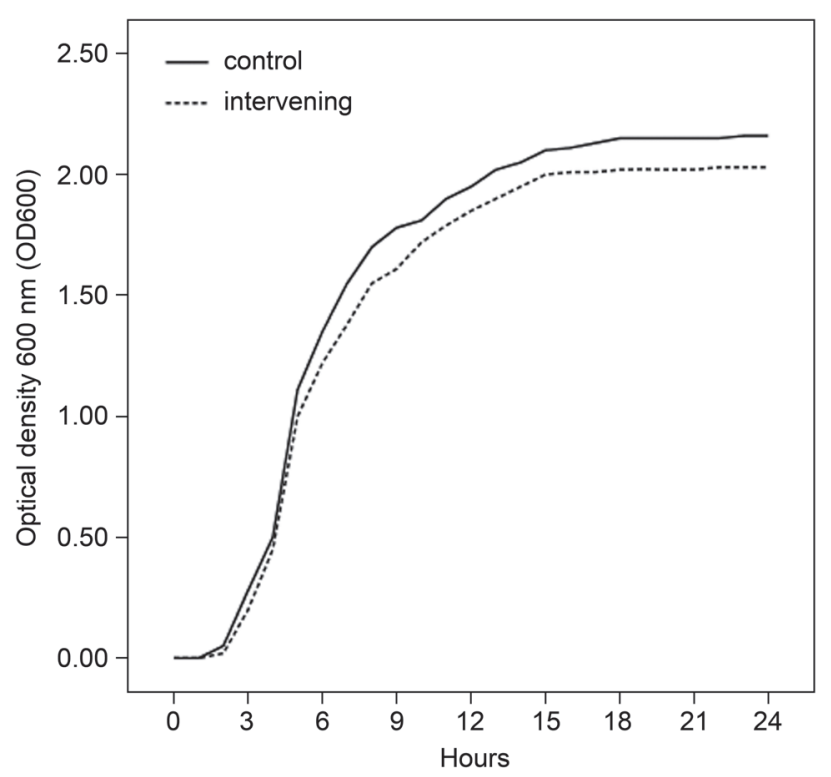

Fig 7. Growth curve of P. aeruginosa PAO1 in saline control group (dotted line) and $128 \mu \mathrm{mol} / \mathrm{l}$ allicin treatment group (solid line).

other hand, had a decreasing trend over time, going from $0.89 \pm 0.01$ on day 1 , to $0.85 \pm 0.02$ on day 3 , and then to $0.76 \pm 0.128$ on day 7 . The ADD increased, from $1.68 \pm 0.13$ on day 1 , to $1.81 \pm 0.22$ on day 3 , and then to $2.54 \pm 0.33$ on day 7 , as did the TE from $5.62 \pm 0.89$ on day 1 , to $6.15 \pm 0.82$ on day 3 , then to $6.78 \pm 0.09$ on day 7. Seven days of biofilm treatment with allicin at the concentration of $128 \mu \mathrm{g} / \mathrm{ml}$ resulted in biofilm thickness being decreased from $28.83 \mu \mathrm{m}$ to $16.50 \mu \mathrm{m}$. Meanwhile, AP was increased from $0.76 \pm 0.128$ to $0.92 \pm 0.02$, and TE was decreased from $6.78 \pm 0.93$ to $5.61 \pm 0.55$ (Fig. 6). These values were significantly different from the control. Allicin treatment at $10 \mu \mathrm{g} / \mathrm{ml}$ produced a similar but remarkably less extensive effect than the high dose of $128 \mu \mathrm{g} / \mathrm{ml}$.

Allicin had a minimal effect on P.aeruginosa PAO1 growth after $\log$ phase. As shown by the growth curve (Fig. 7), the P.aeruginosa PAO1 growth entered a stable phase at $8 \mathrm{~h}$ of culture generated with and without treatment of allicin $(128 \mu \mathrm{g} / \mathrm{ml})$. Administration of allicin did not significantly affect the cell density.

Effect of allicin on the production of QS-controlled virulence factors. This experiment was designed to assess the effect of allicin on the production of QScontrolled virulence factors at sub-MIC concentration.
The QS-controlled virulence factors, including exotoxin A, elastase, pyoverdin and rhamnolipid were assessed after treatment with allicin and compared to levels detected in control cultures. Allicin treatment led to significantly down-regulated expression of exotoxin A and elastase and completely inhibited production of rhamnolipid and pyoverdin (Table II).

\section{Discussion}

Biofilm formation can be divided into several phases. The process begins with the adherence of bacteria to biotic or abiotic solid surfaces. To a certain extent, the pathogenicity of P. aeruginosa is closely related to its ability to form biofilms. The present study showed that the plant-derived organosulfur compound allicin can decrease the adhesion ratio of P. aeruginosa. This result was confirmed by fluorescence microscopy, which suggested that allicin had an inhibitory effect on bacterial adhesion in a concentration-dependent manner. Successful bacteria adhesion is mediated by both attractive and repulsive forces, including exopolysaccharides, electrostatic and hydrophobic interactions, steric hindrance, and hydrodynamic forces (Dunne, 2002). Exopolysaccharides enhance bacteria adherence to catheter surfaces and promote coherence with other bacteria to form colonies, which is the basic unit of a biofilm (Ryder et al., 2007) and the viscoelastic properties of matrix determine the biofilm's structure integrity, resistance to stress and ease of dispension (Lau et al., 2009). In this study, the allicin at the concentration used in this experiment did not show inhibitory effect on the growth of bacteria. Our data revealed that the production of EPS was dramatically decreased by allicin, but the dry weight of bacteria was unaffected. It has been reported that allicin reduces the production of polysaccharide intercellular adhesion, which is the main agglutination agent in the biofilm forming strain S.epidermidis (Cruz-Villalón et al., 2011). Therefore, the effect of allicin on the decreasing bacterial adhesion ratio was partially explained by reducing the production of EPS.

We also investigated effect of allicin on the developmental process of biofilm by monitoring the morphology and structural changes that occurred in response

Table II

Production of QS-controlled virulence factors in response to allicin treatment

\begin{tabular}{|c|c|c|c|c|}
\hline & Saline control group & Allicin, $128 \mu \mathrm{g} / \mathrm{ml}$ & $t$ & $p$ \\
\hline Exotoxin $\mathrm{A}, \mathrm{pg} / \mu \mathrm{l}$ & $19.630 \pm 0.573$ & $6.529 \pm 0.289$ & 57.699 & 0.0001 \\
\hline Elastase, OD & $0.467 \pm 0.003$ & $0.032 \pm 0.001$ & 354.284 & 0.0001 \\
\hline Pyoverdin, g/l & $9325.833 \pm 367.675$ & $7819.167 \pm 111.800$ & 13.582 & 0.0001 \\
\hline Rhamnolipid, g/l & $2.009 \pm 0.063$ & $0.269 \pm 0.009$ & 76.710 & 0.0001 \\
\hline
\end{tabular}


to the allicin treatment. In the saline control group, the thickness of biofilm was found to increase daily; in the first three days, the thickness increased by $11 \mu \mathrm{m}$, and after four days by another $5 \mu \mathrm{m}$. This indicated that during the later stage of biofilm formation, the QS system might inhibit the division of bacteria in order to maintain a reasonable bacterial density, possibly by regulating a set of QS-controlled factors (Bjarnsholt et al., 2005; Nadell et al., 2008; Parsek and Greenberg, 2005). AP is defined as the ratio of void area to total area in the CLSM image, while ADD is the average of the minimum distance from each cluster pixel to the nearest void pixel over all cluster pixels in the image. Both of these values objectively reflect the variation in a biofilm's interstitial pore and nutritional supply during the developmental process of BF. In our study, AP was decreased and ADD was increased as the normal bacterial culture progressed, suggesting that as bacterial density increased, the interstitial space of biofilm became smaller and the supply of oxygen and nutrition for biofilm became restricted. Ultimately, the biofilm became stable. TE is a measure of randomness, indicating the microscale heterogeneity in a biofilm. The increasing TE values that were found in our study of normal biofilm formation indicated that over time the biofilm develops a more complex structure and higher ability to resistant antibiotic agents, whether they be synthetic or host-derived (Dunny et al., 2008; Wimpenny et al., 2000). Allicin treatment produced significant effects on these biofilm features. For example, biofilm thickness, ADD and TE were decreased, while $\mathrm{AP}$ was increased in response to allicin treatment at $128 \mu \mathrm{g} / \mathrm{ml}$. However, at the concentration of $10 \mu \mathrm{g} / \mathrm{mL}$, allicin produced the same effects but to a much less extent. This result revealed that allicin caused the biofilm structure to become loose and simple, which could facilitate an antimicrobial agent's ability to permeate into the biofilm's deeper layer. The allicin-induced decrease in biofilm's heterogeneity might reduce the tolerance of the bacteria to antimicrobial agents. Similar results were found in the $1 \mathrm{~d}$ and $3 \mathrm{~d}$ biofilm models, suggesting that the effect of allicin on the formation of biofilm was consistent at each stage. Since the biofilm's structure at the early stage is relatively simple and bacteria inside the biofilm are not yet quiescent, and remain metabolically active, allicin treatment at this stage would be expected to be more effective.

The QS system is known to play an important role in the infectious process of $P$. aeruginosa, by regulating production of some virulence factors and helping to shape the mature 3D biofilm structure. P. aeruginosa resistance to antimicrobial agents can be increased by 10- to 1000-fold when bacteria grow as polymicrobial colonies, as compared with the planktonic bacteria (Mah and O'Toole, 2001). In P. aeruginosa, a complex
QS system, including las, rhl and Pseudomonas quinolone signal (PQS) (Schertzer et al., 2010), controls the production of many virulence factors including exotoxin A, elastase, rhamnolipid and pyoverdin. Exotoxin $\mathrm{A}$ is a single-chain polypeptide secreted by P. aeruginosa that specifically inhibits protein synthesis both in vitro and in vivo and blocks the immune response of the host. Elastase belongs to the matrix metalloproteinases and can degrade EPS and inactivate some immunological agents. In biofilms, rhamnolipid is necessary for forming channels surrounding the macrocolonies and is required to maintain biofilm architecture (Davey et al., 2003). Pyoverdin acts as the analogue of the QS signaling molecule, controlling the production of several virulence factors and itself, and helping to maintain the biofilm's complex 3D-structure (Pritchard, 2006). Our study showed that allicin could significantly downregulate the expression of exotoxin $A$ and elastase and inhibit production of rhamnolipid and pyoverdin. Allicin's inhibitory effect on QS may modulate the binding of acylated homoserine lactone (AHL) to its cognate receptor, inhibiting the production of the AHL molecule, or inhibiting the expression of some QS gene, which should be verified by further research. In our study, we found that allicin reduced the expression of some virulence factors, which in turn might reduce the antibiotic resistance of $P$. aeruginosa.

In summary, we showed that allicin treatment not only reduced the adhesion ratio of P. aeruginosa, but also inhibited EPS secretion and down-regulates production of some QS-controlled virulence factors. The results imply that allicin can disturb the formation and maturation of $P$. aeruginosa biofilm, and suggest that allicin may represent a promising therapeutic candidate for the management of $P$. aeruginosa biofilms.

\section{Acknowledgments}

This study was supported by a grant from the National Nature Science Foundation of China (No. 30772363). We are grateful to Prof. Haluk Beyenal from the Center of Biofilm Engineering at Montana State University (USA) for supplying the Image Structure Analyzer software.

\section{Literature}

Akiyama H., W.K. Huh, K. Fujii, O. Yamasaki, T. Oono and K. Iwatsuki. 2002. Confocal laser microscopic observation of glycocalyx production by Staphylococcus aureus in vitro. J. Dermatol. Sci. 29: 54-61.

Bartoszko-Tyczkowska A., W. Gaszyński, A. Baranowska and E. Tyczkowska-Sieron 2008. Nosocomial infection control in intesalineive therapy. Anestezjol Intesaline Ter. 40: 232-236.

Benincasa M., A. Abalos, I. Oliveira and A. Manresa. 2004. Chemical structure, surface properties and biological activities of the biosurfactant produced by Pseudomonas aeruginosa LBI from soapstock. Antonie van Leeuwenhoek 85: 1-8. 
Beyenal H., C. Donovan, Z. Lewandowski and G. Harkin. 2004 Three-dimensional biofilm structure quantification. J. Microbiol. Methods 59: 395-413.

Bjarnsholt T., P.Ø. Jensen, T.B. Rasmussen, L. Christophersen, H. Calum, M. Hentzer, H.P. Hougen, J. Rygaard, C. Moser, L. Eberl, N. Høiby and M. Givskov. 2005. Garlic blocks quorum sensing and promotes rapid clearing of pulmonary Pseudomonas aeruginosa infections. Microbiology 151: 3873-3880

Clinical and Laboratory Standards Institute. 2007. Performance Standards for Antimicrobial Susceptibility Testing: $17^{\text {th }}$ Informational Supplement. CLSI document M100-S17. Wayne, Pennsylvania.

Costerton J.W. 2001. Cystic fibrosis pathogenesis and the role of biofilms in persistent infection. Trends. Microbiol. 9: 50-52

Cox C.D. and P. Adams. 1985. Siderophore activity of pyoverdin for Pseudomonas aeruginosa. Infect. Immun. 48: 130-138.

Cruz-Villalón G. and C. Pérez-Giraldo. 2011. Effect of allicin on the production of polysaccharide intercellular adhesin in Staphylococcus epidermidis. J. Appl. Microbiol. 110: 723-728

Davey M.E., N.C. Caiazza and G.A. O'Toole. 2003. Rhamnolipid surfactant production affects biofilm architecture in Pseudomonas aeruginosa PAO1. J. Bacteriol. 185: 1027-1036

Drenkard E. 2003. Antimicrobial resistance of Pseudomonas aeruginosa biofilms. Microbes Infect. 5:1213-1219

DuBois M, K.A. Gilles, J.K. Hamilton, P.A. Rebers and F. Smith. 1956. Colorimetric method for determination of sugars and related substances. Anal. Chem. 28: 350-356

Dunne W.M.Jr. 2002. Bacterial adhesion: seen any good biofilms lately. Clin. Microbiol. Rev. 15: 155-166.

Dunny G.M., T.J. Brickman and M. Dworkin. 2008. Mullticellular behavior in bacteria: communication, cooperation, competition and cheating. BioEssays. 296-298.

Harjai K, R.K. Khandwahaa, R. Mittal, V. Yadav, V. Gupta and S. Sharma. 2005. Effect of $\mathrm{pH}$ on production of virulence factors by biofilm cells of Pseudomonas aeruginosa. Folia. Microbiol (Praha). 50: 99-102.

Kirisits M.J. and M.R. Parsek. 2006. Does Pseudomonas aeruginosa use intercellular signalling to build biofilm communities? Cell Microbiol. 8: 1841-1849.

Koulenti D., T. Lisboa, C. Brun-Buisson, W. Krueger, A. Macor, J. Sole-Violan, E. Diaz, A. Topeli, J. DeWaele, A. Carneiro, I. Martin-Loeches, A. Armaganidis, J. Rello and EU-VAP/CAP Study Group. 2009. Spectrum of practice in the diagnosis of nosocomial pneumonia in patients requiring mechanical ventilation in European intensive care units. Crit. Care. Med. 37: 2360-2368

Kozak K.J. and C.B. Saelinger. 1988. Preparation of Pseudomonas exotoxin A. Methods Enzymol. 165: 147-152.

Lau P.C., J.R. Dutcher, T.J. Beveridge and J.S. Lam. 2009. Absolute quantitation of bacterial biofilm adhesion and viscoelasticity by microbead force spectroscopy. Biophys. J. 96: 2935-2948

Mah T.F. and G.A. O’Toole. 2001. Mechanisms of biofilm resistance to antimicrobial agents. Trends Microbiol. 9: 34-39.
Nadell C.D., J.B. Xavier, S.A. Levin and K.R. Foster. 2008. The evolution of quorum sensing in bacterial biofilms. PLoS. Biol. 6: e14. Ohman D.E., S.J. Cryz and B.H. Iglewski. 1980. Isolation and characterization of Pseudomonas aeruginosa $\mathrm{PAO}$ mutant that produces altered elastase. J. Bacteriol. 142: 836-842.

Parsek M.R. and E.P. Greenberg. 2005. Sociomicrobiology: the connections between quorum sensing and biofilms. Trends Microbiol. 13: 27-33.

Pérez-Giraldo C, G. Cruz-Villalón, R. Sánchez-Silos, R. Martínez-Rubio, M.T. Blanco and A.C. Gómez-García. 2003. In vitro activity of allicin agaist Staphylococcus epidermidis and influence of subinhibitory concentrations on biofilm formation. J. Appl. Microbiol. 95: 709-711.

Petit A.C., N. Noiret, J. Guezennec, N. Gondrexon and S. ColliecJouault. 2007. Ultrasonic depolymerization of an exopolysaccharide produced by a bacterium isolated from a deep-sea hydrothermal vent polychaete annelid. Ultrason Sonochem. 14: 107-112.

Pritchard D.I. 2006. Immune modulation by Pseudomonas neruginosa quorum-sensing signal. Int. J. Med. Microbiol. 296: 111-116 Prithiviraj B., H.P. Bais, T. Weir, B. Suresh, E.H. Najarro, B.V. Dayakar, H.P. Schweizer and J.M. Vivanco. 2005. Down regulation of virulence factors of Pseudomonas aeruginosa by salicylic acid attenuates its virulence on Arabidopsis thaliana and Caenorhabditis elegans. Infect. Immun. 73: 5319-5328

Rasmussen T.B., T. Bjarnsholt, M.E. Skindersoe, M. Hentzer, P. Kristoffersen, M. Köte, J. Nielsen, L. Eberl and M. Givskov. 2005, Screening for quorum-sensing inhibitors (QSI) by use of a novel genetic system, the QSI selector. J. Bacteriol. 187: 1799-1814 Rumbaugh K.P., J.A. Griswold and A.N. Hamood. 2000. The role of quorum sensing in the in vivo virulence of Pseudomonas aeruginosa. Microbes Infect. 2: 1721-1731.

Ryder C, M. Byrd and D.J. Wozniak. 2007. Role of polysaccharides in Pseudomonas aeruginosa biofilm development. Curr. Opin. Microbiol. 10: 644-648.

Schertzer J.W., S.A. Brown, and M. Whiteley. 2010. Oxygen levels rapidly modulate Pseudomonas aeruginosa social behaviours via substrate limitation of PqsH. Mol. Microbiol. 77:1527-1538

Shigematsu T, N. Suda, K. Okuda and J. Fukushima. 2007. Reliable enzyme-linked immunosorbent assay systems for pathogenic factors of Pseudomonas aeruginosa alkaline proteinase, elastase, and exotoxin A: a comparison of methods for labeling detection antibodies with horseradish peroxidase. Microbiol. Immunol. 51: 1149-1159.

Smith A.W. and B.H. Iglewski. 1989. Transformation of Pseudomonas aeruginosa by electroporation. Nucleic Acids Res. 17: 10509 Velliyagounder K., K. Ganeshnarayan, S.K. Velusamy and D.H. Fine. 2012. In vitro efficacy of diallyl sulfides against the periodontopathogen Aggregatibacter actinomycetemcomitans. Antimicrob. Agents Chemother. 56: 2397-2407

Wimpenny J., W. Manz and U. Szewzyk. 2000. Heterogeneity in biofilms. FEMS. Microbiol. Rev. 24: 661 